\title{
Early detection of asthma exacerbations by using action points in self-management
} plans

\author{
Persijn J. Honkoop*\#, D. Robin Taylor", Andrew D. Smith", \\ Jiska B. Snoeck-Stroband* and Jacob K. Sont*
}

ABSTRACT: Our aim was to validate optimal action points in written action plans for early detection of asthma exacerbations.

We analysed daily symptoms and morning peak expiratory flows (PEFs) from two previous studies. Potential action points were based on analysis of symptom scores (standard deviations) percentage of personal best PEF, PEF variability in relation to a run-in period or combinations of these measures. Sensitivity and specificity for predicting exacerbations were obtained for each action point. The numbers needed to treat to prevent one exacerbation and the time interval between reaching action point criteria and the start of the exacerbation were calculated. Based on these parameters, the optimal action points for symptoms, PEF and PEF plus symptoms were determined, and their performance compared with published guidelines' action points.

The optimal action points were, for symptoms, statistical variability (standard deviations) and, for PEF, $<\mathbf{7 0} \%$ of personal best. The combination of PEF plus symptoms performed best, with improved specificity and earlier detection. The main benefits associated with using these action points was to reduce false positive rates for detecting exacerbations.

Early detection of asthma exacerbations can be improved using a composite action point comprising symptoms and PEF measurements over 1 week.

KEYWORDS: Peak flow, prediction, quality control analysis, symptoms

E xacerbations of asthma are common and, even when asthma is mild, constitute a significant health risk [1]. Assessing future risk of adverse events, including exacerbations, and educating patients to use a self-management plan is recommended [2-6].

Self-management includes developing individualised Written Asthma Action Plans (WAAPs). WAAPs specify the level of symptoms or peak expiratory flow (PEF) (called action points, APs) at which to adjust medication (usually starting oral corticosteroids) in order to either prevent or reduce the severity of exacerbations. To ensure effective intervention, an AP should detect an imminent exacerbation well before its onset.

GIBSON et al. [7] and GIBSON and POWELl [8] have previously validated several APs using quality control analysis (QCA). However, in the Global Initiative for Asthma (GINA) guidelines and the Dutch national guidelines, thresholds for symptoms or PEF are not specified [3,9]. Although APs in the current British Thoracic Society (BTS) and US National Heart Lung and Blood Institute (NHLBI) guidelines are more specific, these APs have not been validated $[2,5,6]$. The optimum time point at which changes in either symptoms or PEF may be detected, or the relevant thresholds reached prior to an exacerbation are largely unknown. This lack of validation means that physicians often determine APs for individual patients empirically. If APs are inaccurately selected, this potentially leads to over treatment (false-positive APs) or missed opportunities for early intervention (falsenegative APs).

In this study, our aim was to develop optimal APs based on symptoms and/or PEF threshold levels for early detection of asthma exacerbations that allow timely intervention in patients with mild-tomoderate asthma. Subsequently, we aimed to validate the performance of the optimised APs in a similar but separate study population.
AFFILIATIONS

*Dept of Medical Decision Making, Leiden University Medical Center, and

${ }^{\#}$ Dept of Public Health and Primary Care, Leiden University Medical Center, Leiden, The Netherlands. "Dept of Respiratory Medicine, Dunedin School of Medicine, University of Otago, Dunedin, New Zealand.

CORRESPONDENCE

P.J. Honkoop

Leiden University Medical Center Postzone J-10-S

room J-10-87

Albinusdreef 2

P0 Box 9600

2300 RC Leiden

The Netherlands

E-mail: P.J.Honkoop@lumc.nl

Received:

Nov 242011

Accepted after revision:

March 192012

First published online:

May 312012 


\section{METHODS}

We analysed asthma symptoms, morning PEFs, the occurrence of exacerbations and the use of prednisone using data from written daily diaries from two previous studies [10,11]. The development dataset was obtained from a randomised controlled trial designed to compare the effects of 6 months of treatment with regular inhaled salbutamol, salmeterol or placebo [10]. The validation dataset was obtained from a single-blind placebo-controlled trial that explored the use of exhaled nitric oxide fraction (FeNO) to guide treatment in chronic asthma [11]. The follow-up period was $1 \mathrm{yr}$.

\section{Subjects}

There were 165 patients in the development dataset and 94 in the validation dataset, all with stable mild-to-moderate chronic asthma $[10,11]$.

\section{Daily diaries}

In both studies, daily diary recordings included symptoms of daytime and night-time chest tightness/wheeze/dyspnoea, cough, sputum production, exercise impairment, and either appearance of or increased frequency of nocturnal awakening. All were scored on a $0-3$ scale or by a yes/no response where appropriate. The best of three PEF measurements was also recorded each morning and evening. Missing data were interpolated using the mean of the recordings from the previous and following days.

\section{Exacerbations}

Exacerbations were defined in both studies using a composite daily asthma score. The scoring criteria were similar between the two studies, but differed regarding the use of a $\beta$-agonist "reliever" and nocturnal awakening (table 1 in TAYLOR et al. [10] and table 2 in SMITH et al. [11]). In brief, major exacerbations were defined as a visit to the emergency department, a PEF $<40 \%$ personal best $(\mathrm{pb})$ for $\geqslant 1$ day, a PEF $<60 \%$ pb for $\geqslant 2$ days plus an increase in symptoms, or a PEF $<60 \%$ pb for $\geqslant 1$ day and PEF $<75 \%$ pb for $\geqslant 2$ days with an increase in symptoms.

During the study, courses of prednisone were administered in response to deteriorating symptoms and/or peak flows, or at the discretion of patients or clinicians independently of diary data. Prednisone use for $\geqslant 3$ days is widely used as a definition for exacerbations [4]. Therefore, as a sensitivity analysis, we also assessed the predictive utility of APs using this alternative definition.

\section{Action points}

A range of pre-specified APs was evaluated. For symptoms, we assessed APs used in currently recommended WAAPs: the occurrence of nocturnal awakening or the appearance of any symptoms [2, 3, 6, 9]. Additionally, we evaluated APs based on QCA of symptoms using standard deviations from the mean symptom score during run-in for each patient. To this end, we developed a composite daily symptom score (range 0-6), which combined all daily recorded individual symptoms and "reliever" $\beta$-agonist use, with higher scores representing more severe symptoms (table S5). The mean score and its standard deviation were determined per patient during the run-in period when asthma was well controlled. Subsequently, occasions characterised by deviation from the mean by more than one, two or three standard deviations were evaluated as potential APs. In patients without any symptoms during the run-in, the mean symptom score and standard deviation was 0 . In these cases, the one, two and three standard deviation thresholds were set at $0.17,0.34$ and 0.50 , respectively, representing the minimal possible changes in composite symptom score.

For PEF, the APs were derived from percentages of personal best morning PEF measurement obtained during the run-in period (\% pb), or QCA based on the approach outlined by GIBSON et al. [7] and GIBSON and Powell [8]. We also analysed whether combining PEF and symptoms as a composite AP might perform better, since using single outliers of PEF or symptoms alone might result in relatively high false positive rates for exacerbation prediction. Therefore, we assessed whether a combination of symptom and PEF thresholds were reached on the same day, and also within a 1-week time window. Finally, we assessed the performance of the APs currently recommended by the NHLBI, which are based on both symptoms and PEF ("yellow zone") [2]. As it is not clear whether reaching the threshold for either symptoms or PEF alone is sufficient or both are required, we analysed both options.

For each patient, every week in the diary recordings was coded as either a "stable week", when no exacerbation occurred, or "pre-exacerbation week' for the week prior to an exacerbation. For all stable and pre-exacerbation weeks, we assessed whether the $\mathrm{AP}(\mathrm{s})$ either predicted a future exacerbation (when one or more of the daily recordings in that week fulfilled criteria for that specified AP), or predicted that a future exacerbation would not occur (when daily recording(s) did not reach the defined thresholds) (fig. 1).

\section{Analysis}

All analyses were performed with STATA (release 11; StataCorp, College Station, TX, USA). Contingency tables for each AP threshold were constructed to calculate performance characteristics including sensitivity, specificity, accuracy and area under the receiver operating characteristic (AUC) curve for predicting an exacerbation. In addition, for each AP threshold we assessed the (potential) number needed to treat (NNT) in order to prevent one exacerbation, given a hypothetical perfect treatment and early detection, defined as the number of days before the onset of an exacerbation the AP was reached for the first time in a preexacerbation week. NNT was calculated by dividing the total number of times an AP was reached (true positives and false positives) by the number of times it accurately predicted a future exacerbation (true positives).

The APs that performed optimally were grouped within four categories: 1) symptoms solely; 2) PEF solely; 3) symptoms and PEF on the same day; and 4) symptoms and PEF within 1 week prior to an exacerbation, using the development dataset. Optimal performance was defined as a sensitivity of $\geqslant 75 \%$ combined with the best trade-off between early detection and potential NNT. To determine this outcome, we plotted the number of days on which an exacerbation was predicted before its occurrence against the NNT for a series of different APs (fig. 2).

To assess the external validity of the optimal APs derived from the development dataset, their performance was assessed and compared with several published APs using the validation dataset [11]. 


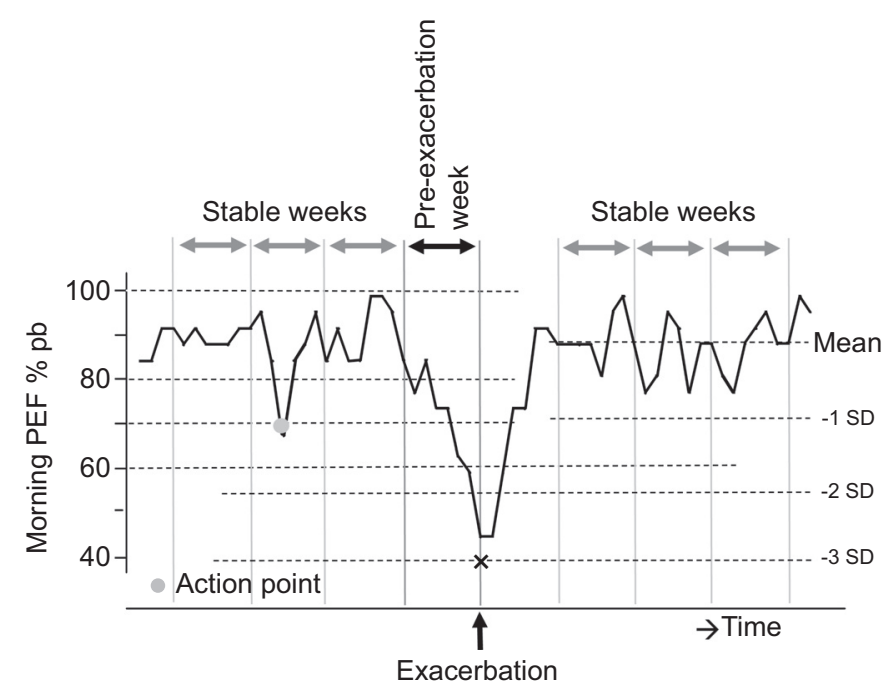

FIGURE 1. The use of action points in an 8-week peak flow chart with an exacerbation at the half-way point. The dotted lines indicate the thresholds of potential action points, on the left based on \% of personal best ( $\mathrm{pb}$ ) peak expiratory flow (PEF), and on the right based on individual standard deviations for PEF. The observation period is divided into weeks before and during the exacerbation, and weeks of normal control, respectively coded as pre-exacerbation weeks and stable weeks. In this example, we have highlighted the action points $\mathrm{PEF}<70 \% \mathrm{pb}$ and $\mathrm{PEF}$ $<-3 \mathrm{SD}$. The action point PEF $<70 \% \mathrm{pb}$ is reached twice, once as a false positive in a stable week and once accurately 2 days before the exacerbation in the preexacerbation week. The action point PEF $<-3$ SD is never reached in this example, representing a false negative prediction for the pre-exacerbation week (marked X).

\section{RESULTS}

The development dataset consisted of daily recordings from 164 patients. 88 exacerbations, defined using diary data, occurred during 18 months of follow-up. Exacerbations occurred in 39 different patients, a mean rate 1.8 per patient per year, ranging from 1 to 13.147 exacerbations, defined as the use of a course of oral prednisone, occurred during the follow-up interval.

In the validation dataset, 94 patients provided daily recordings. 22 exacerbations occurred. Exacerbations occurred in 17 patients and the mean rate was 1.5 per patient per year (range 1 to 5). Oral prednisone was used on 75 occasions.

The characteristics of patients from both studies are listed in table 1.

\section{Action points}

The performance of 25 potential APs was analysed (a complete overview of results is presented in tables S4a-d). Six APs were based on symptoms, eight on PEF, nine on combinations of symptoms and PEF on the same day, and two on combinations of symptoms and PEF within 1 week. In general, APs based on standard deviations of symptom scores performed better than pre-defined absolute levels of symptoms. This judgment was based on lower NNTs for the former approach. PEF using \% pb resulted in considerably lower NNTs than using standard deviations.

The optimal symptom AP was a score that increased by more than two standard deviations more than the run-in mean, and this detected exacerbations 2.9 days before occurrence with $88.5 \%$ sensitivity, $86.3 \%$ specificity and a NNT of 24 . For PEF, the optimally performing $\mathrm{AP}$ was a $\mathrm{PEF}<60 \% \mathrm{pb}$, which is also currently proposed by the BTS as the threshold for commencing oral prednisone treatment [5]. It had a sensitivity of $78.2 \%$, specificity of $98.7 \%$ and a NNT of 3 . However, it detected exacerbations only 1 day before their occurrence. The optimal combination (symptoms and PEF) comprised a symptom score increase of more than two standard deviations plus PEF decrease to $<70 \% \mathrm{pb}$. This combination detected exacerbations
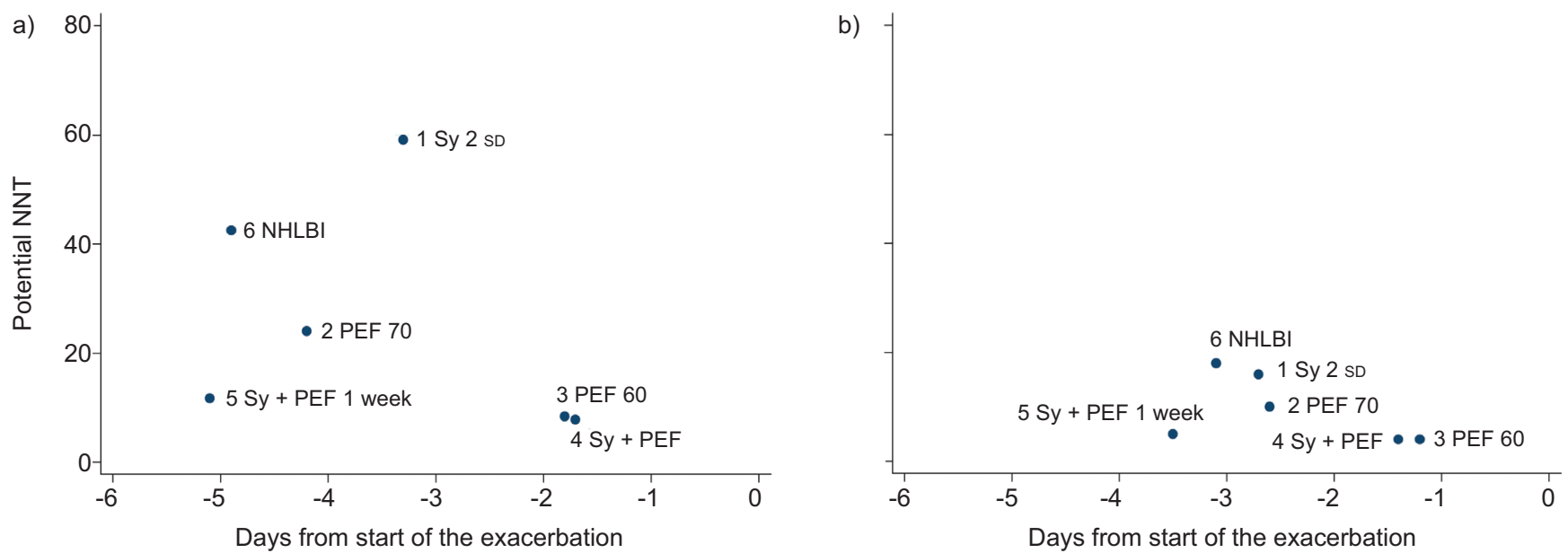

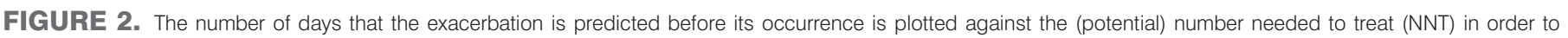

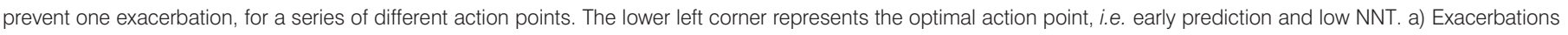

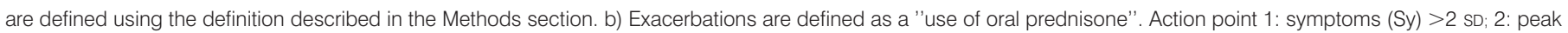

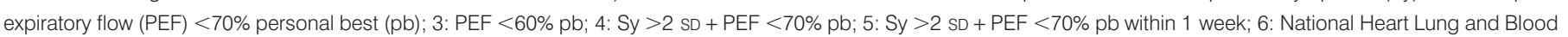

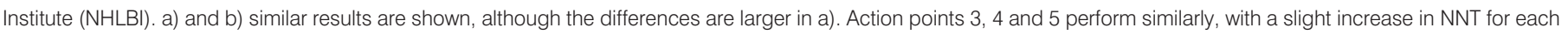

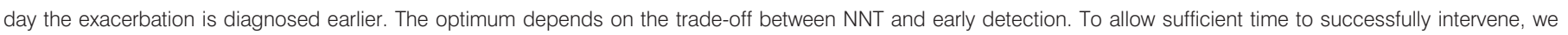
opted for number 5. Action points 1,2 and 6 perform considerably worse, due to the high NNTs. 


\begin{tabular}{|c|c|c|}
\hline \multirow[t]{2}{*}{ TABLE 1} & \multirow[b]{2}{*}{$\begin{array}{l}\text { Development } \\
\text { dataset [10] }\end{array}$} & \multirow[b]{2}{*}{$\begin{array}{l}\text { Validation } \\
\text { dataset [11] }\end{array}$} \\
\hline & & \\
\hline Patients n & 164 & 94 \\
\hline Age yrs & $38(18-64)$ & $44(12-73)$ \\
\hline \multicolumn{3}{|l|}{ Sex } \\
\hline Male & $73(45)$ & $35(37)$ \\
\hline Female & 91 (55) & 59 (63) \\
\hline \multicolumn{3}{|l|}{ Taking regular $\mathrm{ICS}^{\#}$} \\
\hline None & 8 & 0 \\
\hline $1-400 \mu \mathrm{g} \cdot \mathrm{day}^{-1}$ & 34 & 53 \\
\hline $401-1000 \mu \mathrm{g} \cdot$ day $^{-1}$ & 36 & 45 \\
\hline$>1000 \mu \mathrm{g} \cdot$ day $^{-1}$ & 22 & 2 \\
\hline $\begin{array}{l}\text { Symptom score during } \\
\text { run-in period }\end{array}$ & $0.55(0-2.06)$ & $0.56(0-2.65)$ \\
\hline $\begin{array}{l}\text { Personal best PEF during } \\
\text { run-in } L \cdot \mathrm{min}^{-1}\end{array}$ & $508(305-755)$ & $448(230-705)$ \\
\hline
\end{tabular}

Data are presented as mean (range), $\mathrm{n}(\%)$ or \%, unless otherwise stated. ICS: inhaled corticosteroids; PEF: peak expiratory flow. \#: beclomethasone equivalent; ${ }^{\bullet}$ : maximum score of 6.0 .

1.4 days before their occurrence with $80.5 \%$ sensitivity, $98.3 \%$ specificity and a NNT of 4 . Within a 1-week window, this symptom-PEF combination detected exacerbations 4.1 days (mean) before their occurrence with a sensitivity of $85.1 \%$, specificity of $97.2 \%$ and a NNT of 6 (table 2).
The performance characteristics of optimal APs in the validation dataset are presented in table 3. In general, the sensitivities for each of the optimal APs differed somewhat from those obtained using the developmental dataset, whereas specificities remained similar. For each optimal AP, the number of days before the onset of an exacerbation at which the AP predicted future exacerbations was better in the validation dataset, i.e. between 0.4 and 1.0 day earlier.

For both versions of the AP recommended by the NHLBI, the combination of "appearance of any symptoms" plus PEF $<80 \%$ pb performed best (table 3). It detected exacerbations 4.9 days before onset, with a sensitivity of $100 \%$ and specificity of $86.8 \%$. However, the NNT is 43 , whereas it is 12 for the optimal AP from the development dataset (fig. 2).

The comparable data using the alternative definition of "use of oral prednisone" are also reported in tables 2 and 3, and tables S4a-d in online supplementary material. In general, sensitivities were considerably lower, overall accuracies were similar, early diagnosis was slightly later, but the NNTs were better.

\section{DISCUSSION}

The present study provides the most comprehensive data to date of the performance characteristics of a range of symptom and/or PEF thresholds at which patients might intervene to abort an asthma exacerbation or to reduce its severity. For symptoms, a change of more than two standard deviations in a composite symptom score provided optimum outcomes. For PEFs, a decrease to $<60 \% \mathrm{pb}$ was optimal. However, an AP based on a combination of changes in symptom score (more than two standard deviations) and PEF ( $<70 \%$ pb) occurring during a 1week period performed even better. This combination predicted

TABLE 2 Performance characteristics in the development dataset of the optimal action points (APs) per category

\begin{tabular}{|c|c|c|c|c|c|c|c|}
\hline $\begin{array}{l}\text { AP category and } \\
\text { optimal criteria }\end{array}$ & $\begin{array}{l}\text { Definition of } \\
\text { exacerbation }\end{array}$ & $\begin{array}{l}\text { Early detection } \\
\text { days }\end{array}$ & $\begin{array}{c}\text { Sensitivity } \\
\%\end{array}$ & $\begin{array}{c}\text { Specificity } \\
\%\end{array}$ & $\begin{array}{c}\text { Accuracy }{ }^{\top} \\
\%\end{array}$ & $\mathrm{AUC}^{+}$ & $N N T^{s}$ \\
\hline Symptoms >2 SD & Use of prednisone & 2.7 & 76.9 & 86.6 & 86.5 & 0.82 & 17 \\
\hline \multirow[t]{2}{*}{ PEF $<70 \%$ pb } & Symptoms PEFs & 2.9 & 90.8 & 93.9 & 93.9 & 0.92 & 11 \\
\hline & Use of prednisone & 2.6 & 61.2 & 93.9 & 93.5 & 0.78 & 10 \\
\hline \multirow{2}{*}{$\begin{array}{c}\text { Symptoms + PEF: } \\
\text { same day }\end{array}$} & Symptoms PEFs & 1.4 & 80.5 & 98.3 & 98.2 & 0.89 & 4 \\
\hline & Use of prednisone & 1.4 & 47.6 & 98.3 & 97.7 & 0.72 & 4 \\
\hline \multirow{2}{*}{$\begin{array}{l}\text { Symptoms + PEF: } \\
\text { within } 1 \text { week }\end{array}$} & Symptoms PEFs & 4.1 & 85.1 & 97.2 & 97.1 & 0.91 & 6 \\
\hline & Use of prednisone & 3.5 & 54.4 & 97.2 & 96.7 & 0.76 & 5 \\
\hline
\end{tabular}

AUC: area under the receiver operating characteristic curve; NNT: number needed to treat; PEF: peak expiratory flow; pb: personal best. ${ }^{\#}$ : a description of how many days before the onset of an exacerbation this AP will predict the future occurrence of the event. It was assessed by calculating the mean number of days that this AP's thresholds were reached for the first time in the week preceding the exacerbation, from all predicted exacerbations. ": ability of this AP to correctly predict an exacerbation and how often exacerbations are missed or falsely predicted. ${ }^{+}:$a measure of the overall accuracy of a prediction, with 1.0 representing a perfect prediction with $100 \%$ sensitivity and 100\% specificity, and 0.5 representing a random guess and, therefore, the model has no predictive properties. In general, AUC values of $<0.7$ do not have clinical significance. ${ }^{\text {s: }}$ the number of times this AP is positive per predicted exacerbation. It is a measure of how often an intervention is applied unnecessarily to prevent

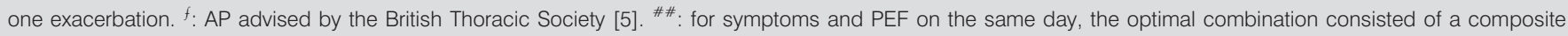
symptom score $>2$ SD of the mean plus PEF $<70 \%$ of pb. ${ }^{~} "$ : for symptoms and PEF within 1 week the optimal combination consisted of a composite symptom score $>2$ SDS of the run-in mean plus PEF $<70 \%$ of pb, with a 7 -day time window being allowed for either threshold to become positive. 
exacerbations 5 days before their occurrence, thus allowing sufficient time to intervene, whilst the NNT remained low.

Previously, in a Cochrane review, POWELL and GIBSON [12] compared the use of WAAPs based on symptoms with those based on PEF [12]. Results showed that these were equivalent with regard to outcomes, i.e. hospitalisations or unscheduled doctor visits. Our data indicate that combining symptoms and PEFs provide added value. Clearly, it is not practical for patients to do the necessary calculations and therefore, in practice, an AP based solely on PEF $<60 \%$ pb might be optimal. Nevertheless, with the advent of internet-based applications ("Apps"), the use of seemingly complex APs is now feasible [13]. Although compliance with paper diary recordings is generally poor [14], such an approach is feasible with electronic recordings [15] and is of particular relevance in patients with difficult or brittle asthma.

The fact that a "both/and" combination of symptoms and PEF performed better than single APs is not surprising. Even with good asthma control, symptoms and PEFs may vary discordantly, and one of these parameters may change in isolation, especially in "poor perceivers". APs with threshold levels based solely on either symptoms or PEF are susceptible to these variations. Using a more stringent threshold, such as PEF $<60 \%$ $\mathrm{pb}$, can solve this issue, but has the disadvantage of late detection of an imminent exacerbation. Therefore, using a 1week window for the symptoms plus PEF provided the best AP as it detected exacerbations 5.1 days before occurrence, at only a slight cost in specificity and NNT. To assess whether symptoms or PEF drive earlier detection using the AP with a one week time window, we performed a subgroup analysis of the 74 predicted exacerbations. There was no consistent pattern as to whether changes in symptoms preceded PEFs or vice versa. Symptoms occurred earlier in 25 subjects, the threshold for PEF changes was reached earlier in 23 , and in 26 there was no discordance.

Previously, GiBSON et al. [7] analysed nine different APs and showed that QCA of daily PEFs performs better than percentages of personal best PEF (in contrast to the present data) or percentage predicted of PEF. GIBSON et al. [7] reported that the optimal QCA AP detected 91\% of exacerbations and falsely predicted an exacerbation in $23 \%$ of periods of normal control. TATTERSFIELD et al. [16] analysed the false positive rate of APs based on the median values of PEF and symptoms at 2 days before the start of an exacerbation. They found a false-positive rate of $6.4 \%$ using the advent of night-time symptoms, $26 \%$ for morning PEF and $30 \%$ for daytime symptoms. THAMRIN et al. [17] analysed daily fluctuations in PEF and, by calculating conditional probabilities of future decreases in lung function, predicted the risk of exacerbations with a sensitivity of $68.8 \%$ and specificity of $67.4 \%$. The AUC was 0.85 , which is only slightly lower than AUCs of most optimal APs in this study [17].

The time course of changes in symptoms and PEF that constitute an asthma exacerbation is important in determining the optimum time for intervention. If changes can only be identified after the time at which intervention is likely to be effective, then the rationale for using WAAPs would be weak. Previous data suggest that symptoms and PEF start declining 5-10 days before exacerbations [16, 18]. The changes in PEFs

TABLE 3 Performance characteristics in the validation dataset of optimal action points (APs) derived from the development dataset and of the National Heart Lung and Blood Institute (NHLBI) AP

\begin{tabular}{|c|c|c|c|c|c|c|c|}
\hline AP category and optimal criteria & $\begin{array}{l}\text { Definition of } \\
\text { exacerbation }\end{array}$ & $\begin{array}{l}\text { Early detection }{ }^{\#} \\
\text { days }\end{array}$ & $\begin{array}{c}\text { Sensitivity } \\
\%\end{array}$ & $\begin{array}{c}\text { Specificity } \\
\%\end{array}$ & $\begin{array}{c}\text { Accuracy } \\
\%\end{array}$ & $\mathrm{AUC}^{+}$ & $\mathrm{NNT}^{\S}$ \\
\hline \multirow[t]{2}{*}{ Symptoms $>2$ SD } & Symptoms PEFs & 3.3 & 75.0 & 86.2 & 86.2 & 0.81 & 59 \\
\hline & Use of prednisone & 4.1 & 60.0 & 86.9 & 86.6 & 0.73 & 19 \\
\hline \multirow[t]{2}{*}{ PEF $<70 \%$ pb } & Symptoms PEFs & 4.2 & 100 & 92.5 & 92.6 & 0.96 & 24 \\
\hline & Use of prednisone & 3.6 & 53.3 & 93.0 & 92.5 & 0.73 & 12 \\
\hline \multirow[t]{2}{*}{ PEF $<60 \% \mathrm{pb}^{f}$} & Symptoms PEFs & 1.8 & 100 & 97.6 & 97.6 & 0.99 & 8 \\
\hline & Use of prednisone & 2.3 & 18.7 & 97.5 & 96.6 & 0.55 & 12 \\
\hline \multirow[t]{2}{*}{ Symptoms + PEF: same day } & Symptoms PEFs & 1.7 & 75.0 & 98.3 & 98.2 & 0.87 & 8 \\
\hline & Use of prednisone & 2.1 & 29.3 & 98.5 & 97.6 & 0.64 & 5 \\
\hline \multirow[t]{2}{*}{ Symptoms + PEF: within 1 week } & Symptoms PEFs & 5.1 & 75.0 & 97.4 & 97.3 & 0.86 & 12 \\
\hline & Use of prednisone & 4.8 & 33.3 & 97.6 & 96.8 & 0.65 & 7 \\
\hline \multirow{2}{*}{$\begin{array}{l}\text { NHLBI criteria changes in symptoms } \\
\text { and in PEF }{ }^{\# \#}\end{array}$} & Symptoms PEFs & 4.9 & 100 & 86.8 & 86.9 & 0.93 & 43 \\
\hline & Use of prednisone & 4.0 & 70.7 & 87.5 & 87.3 & 0.79 & 16 \\
\hline \multirow{2}{*}{$\begin{array}{l}\text { NHLBI criteria changes in either } \\
\text { symptoms or in PEF }\end{array}$} & Symptoms PEFs & 6.5 & 100 & 47.5 & 47.6 & 0.74 & 176 \\
\hline & Use of prednisone & 6.1 & 100 & 48.2 & 48.8 & 0.74 & 46 \\
\hline \multicolumn{8}{|c|}{ 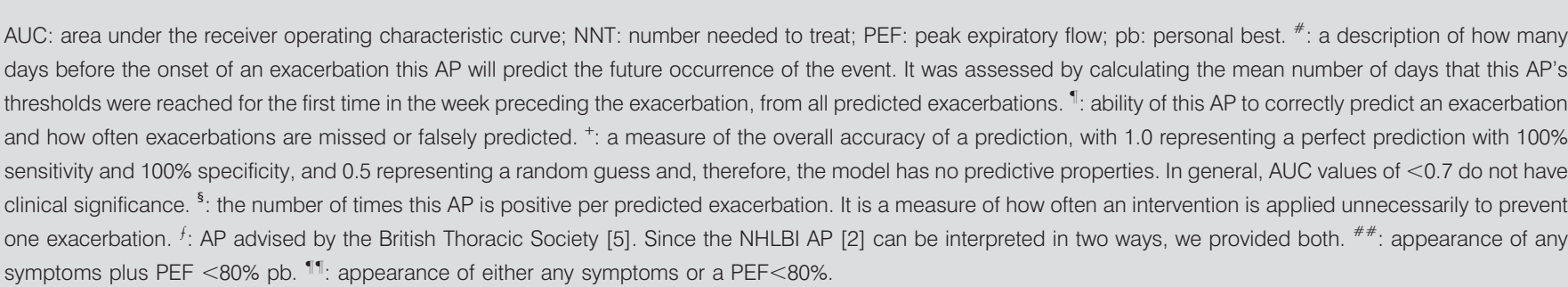 } \\
\hline
\end{tabular}



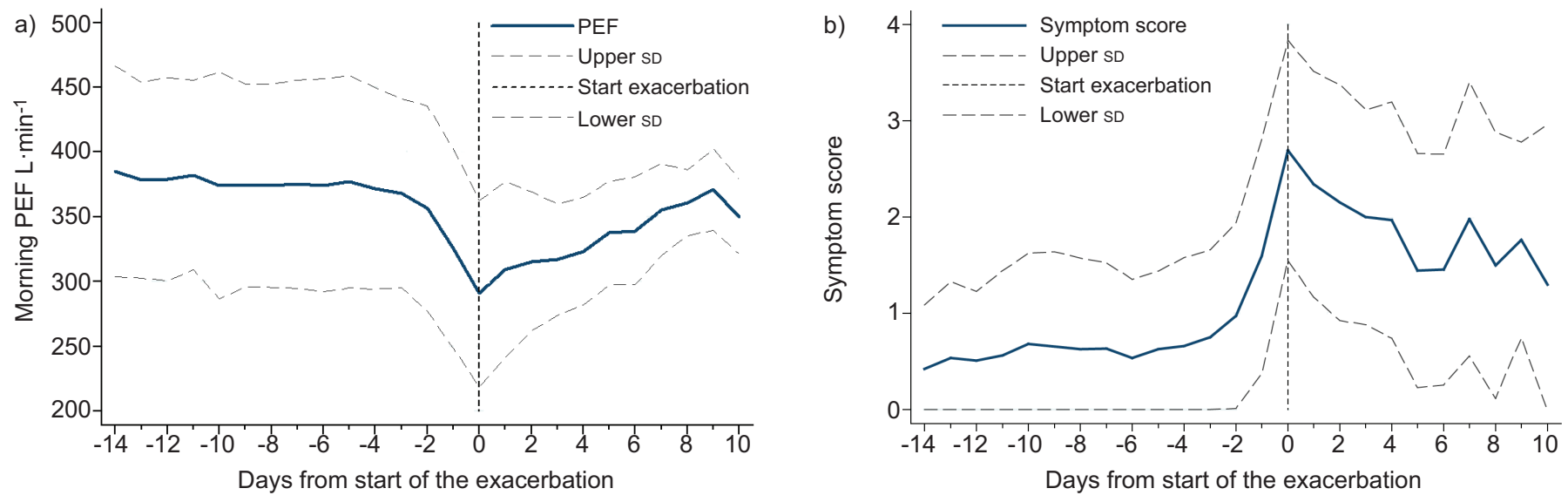

FIGURE 3. a) Changes in peak expiratory flow (PEF) and b) symptom scores from day -14 to day +10 before and after an exacerbation using the mean PEF and symptom score data from each exacerbation in the development dataset.

and symptom scores associated with exacerbations in our patients are illustrated in figure $3 a$ and $b$. Based on these findings, we systematically analysed the 7-day period preceding exacerbations. We found that changes in the optimal APs occurred between 1.7 and 5.1 days before the defined onset of an exacerbation (table S4). The onset of action of systemic corticosteroid is within $12-24 \mathrm{~h}$, and so the APs would be reached in sufficient time to allow for steroids to have a modifying effect. The effectiveness of quadrupling the dose of inhaled corticosteroids was recently investigated by ОвORNE et al. [19], and might have resulted in greater clinical benefits if commenced at the times calculated to be optimal in our study.

Our study has several possible limitations. First, we selected criteria for acceptable sensitivity and specificity (see Statistical analysis section), as we aimed to balance early detection of exacerbations against potential overdiagnosis. Secondly, the composite symptom score(s) used in the two studies were not externally validated. It is not certain whether applying QCA to alternative scoring systems such as the Asthma Control Questionnaire or the Asthma Control Test would give similar results $[20,21]$. However, given the overall similarity between results using both of our datasets, there is reason to believe that QCA is a valid approach to optimising APs independently of the exact scoring system used. Thirdly, APs were based on parameters that were incorporated in the definition of an exacerbation. Our study was not designed to be explanatory but rather to model predictive performance, and as such is methodologically sound. Our definition of major exacerbations, i.e. either emergency room visits or changes in PEF plus symptoms for $\geqslant 2$ days, is in accordance with recent criteria for severe exacerbations [4]. Furthermore, in modified forms, our definition has been used in several previous studies [10, 11, 22, 23]. However, accepting that the definition of an exacerbation is important in the interpretation of our data, we performed additional analyses using "use of oral prednisone" as the definition of an exacerbation (tables 2 and 3 and table S4 of the online supplementary material). The order of optimal APs was similar with regard to early detection and NNT (fig. 2b). Using this definition, the sensitivity to detect exacerbations was considerably lower when using PEF either solely or in combination with symptoms, whereas it was only slightly lower using symptoms alone (table S4). This implies that the decision to administer prednisone depended more on symptoms than on PEF. Given that the sensitivity was $<75 \%$, and the NNT was high, we concluded that the analysed APs did not perform well enough to predict exacerbations defined as "use of oral prednisone. Such events were generally less severe than the exacerbations defined a priori using composite symptom scores and PEFs. It is therefore arguable that our APs performed well in predicting events of higher severity and in which earlier intervention is clinically desirable.

In conclusion, the optimal AP for the early detection of asthma exacerbations consists of a greater than two standard deviations increase in a composite symptom score and a fall in PEF to $<70 \% \mathrm{pb}$, occurring within a 1-week window. With the advent of handheld computer technology, there is potential to use these criteria more readily in day-to-day practice, and thus reduce the impact of exacerbations, particularly in patients with a history of frequent exacerbations. Prospective studies or further analyses using other published datasets should be carried out to confirm the present findings, and together they should be used to revise and improve the empirical recommendations offered in current guidelines.

\section{SUPPORT STATEMENT}

The study was partly funded by a short-term research fellowship awarded by the Netherlands Asthma Foundation.

\section{STATEMENT OF INTEREST}

Statements of interest for A.D. Smith and J.K. Sont can be found at www.erj.ersjournals.com/site/misc/statements.xhtml

\section{ACKNOWLEDGEMENTS}

The developmental and validation datasets were provided to the principal authors (P.J. Honkoop and J.K. Sont), with full permission to undertake additional analyses, by D.R. Taylor.

\section{REFERENCES}

1 O'Byrne PM, Parameswaran K. Pharmacological management of mild or moderate persistent asthma. Lancet 2006; 368: 794-803.

2 National Heart Lung and Blood Institute. National Asthma Education and Prevention Program (NAEPP) Expert panel report 3. 
Guidelines for the diagnosis and management of asthma. Bethesda, NHLBI, 2007.

3 Global strategy for asthma management and prevention. Global initiative for asthma. 2006. www.who.int/respiratory/asthma/ GINA_WR_2006_copyright $\% 5 b 1 \% 5 \mathrm{~d}$.pdf Date last accessed: October 31, 2012.

4 Reddel HK, Taylor DR, Bateman ED, et al. An official American Thoracic Society/European Respiratory Society statement: asthma control and exacerbations. Am J Respir Crit Care Med 2009; 180: 59-99.

5 British Thoracic Society, Scottish Intercollegiate Guidelines Network. British Guideline on the management of Asthma. A national clinical guideline. London, British Thoracic Society, 2008.

6 The Asthma and Respiratory Foundation of New Zealand. Asthma Self-management Plan 2010. Wellington, The Asthma and Respiratory Foundation of New Zealand.

7 Gibson PG, Wlodarczyk J, Hensley MJ, et al. Using quality-control analysis of peak expiratory flow recordings to guide therapy for asthma. Ann Intern Med 1995; 123: 488-492.

8 Gibson PG, Powell H. Written action plans for asthma: an evidencebased review of the key components. Thorax 2004; 59: 94-99.

9 The Dutch General Practice Society (NHG) guideline. Asthma in adults. 2007. http://nhg.artsennet.nl/kenniscentrum/k_richtlijnen/ k_nhgstandaarden/Samenvattingskaartje-NHGStandaard/M27_svk. htm Date last accessed: October 31, 2012.

10 Taylor DR, Town GI, Herbison GP, et al. Asthma control during long-term treatment with regular inhaled salbutamol and salmeterol. Thorax 1998; 53: 744-752.

11 Smith $\mathrm{AD}$, Cowan JO, Brassett KP, et al. Use of exhaled nitric oxide measurements to guide treatment in chronic asthma. $N$ Engl J Med 2005; 352: 2163-2173.

12 Powell H, Gibson PG. Options for self-management education for adults with asthma. Cochrane Database Syst Rev 2003; 1: CD004107.
13 van der Meer V, Bakker MJ, Van Den Hout WB, et al. Internetbased self-management plus education compared with usual care in asthma: a randomized trial. Ann Intern Med 2009; 151: 110-120.

14 Reddel HK. Peak flow monitoring in clinical practice and clinical asthma trials. Curr Opin Pulm Med 2006; 12: 75-81.

15 van der Meer V, Rikkers-Mutsaerts ER, Sterk PJ, et al. Compliance and reliability of electronic PEF monitoring in adolescents with asthma. Thorax 2006; 61: 457-458.

16 Tattersfield AE, Postma DS, Barnes PJ, et al. Exacerbations of asthma: a descriptive study of 425 severe exacerbations. The FACET International Study Group. Am J Respir Crit Care Med 1999; 160: 594-599.

17 Thamrin C, Zindel J, Nydegger R, et al. Predicting future risk of asthma exacerbations using individual conditional probabilities. J Allergy Clin Immunol 2011; 127: 1494-1502.

18 Chan-Yeung M, Chang JH, Manfreda J, et al. Changes in peak flow, symptom score, and the use of medications during acute exacerbations of asthma. Am J Respir Crit Care Med 1996; 154: 889-893.

19 Oborne J, Mortimer K, Hubbard RB, et al. Quadrupling the dose of inhaled corticosteroid to prevent asthma exacerbations: a randomized double blind, placebo controlled, parallel group, clinical trial. Am J Respir Crit Care Med 2010; 180: 598-602.

20 Juniper EF, Svensson K, Mörk AC, et al. Measurement properties and interpretation of three shortened versions of the asthma control questionnaire. Respir Med 2005; 99: 553-558.

21 Nathan RA, Sorkness CA, Kosinski M, et al. Development of the asthma control test: a survey for assessing asthma control. J Allergy Clin Immunol 2004; 113: 59-65.

22 Frey U, Brodbeck T, Majumdar A, et al. Risk of severe asthma episodes predicted from fluctuation analysis of airway function. Nature 2005; 438: 667-670.

23 Taylor DR, Drazen JM, Herbison GP, et al. Asthma exacerbations during long term $\beta$-agonist use: influence of $\beta 2$ adrenoceptor polymorphism. Thorax 2000; 55: 762-767. 